\title{
DETERMINATION OF THE TEMPERATURE FIELD IN BURNED AND HEALTHY SKIN TISSUE USING THE BOUNDARY ELEMENT METHOD - PART II
}

\author{
Katarzyna Freus ${ }^{1}$, Sebastian Freus ${ }^{2}$ \\ ${ }^{1}$ Institute of Mathematics, Czestochowa University of Technology \\ ${ }^{2}$ Institute of Computer and Information Science, Czestochowa University of Technology \\ Częstochowa, Poland \\ ${ }^{1}$ katarzyna.freus@im.pcz.pl, ${ }^{2}$ sebastian.freus@icis.pcz.pl
}

\begin{abstract}
In the paper, the position of the boundary between burned and healthy tissue is described by the NURBS curve. The temperature field in the domain is calculated by means of the boundary element method. The influence of discretization on the temperature distribution in the burned and healthy skin tissue is analysed. Different numbers of boundary elements and internal cells are taken into account. In the final part of the paper the examples of computations are shown.
\end{abstract}

Keywords: bioheat transfer, Pennes equation, boundary element method

\section{Description of boundary}

The domain of burned $\left(\Omega_{1}\right)$ and healthy tissue $\left(\Omega_{2}\right)$, as shown in Figure 1, is considered.

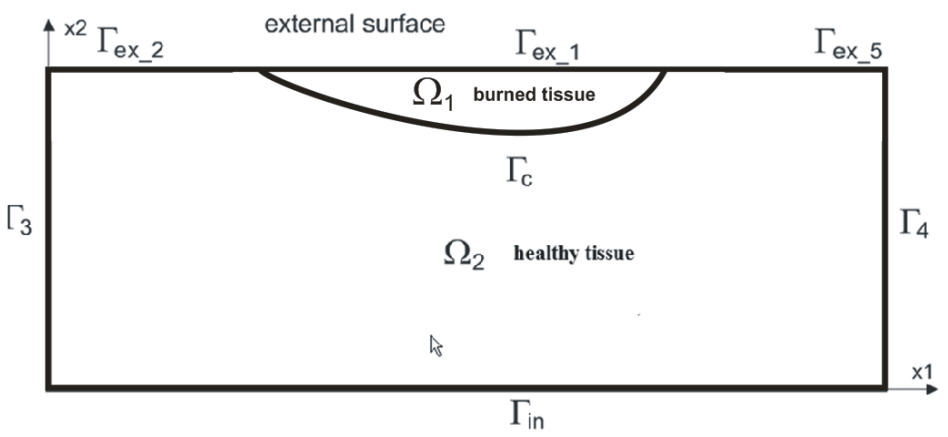

Fig. 1. Domain considered

The temperature distribution in the domains is described by the system of two Pennes equations supplemented by the different boundary conditions [1-4] 


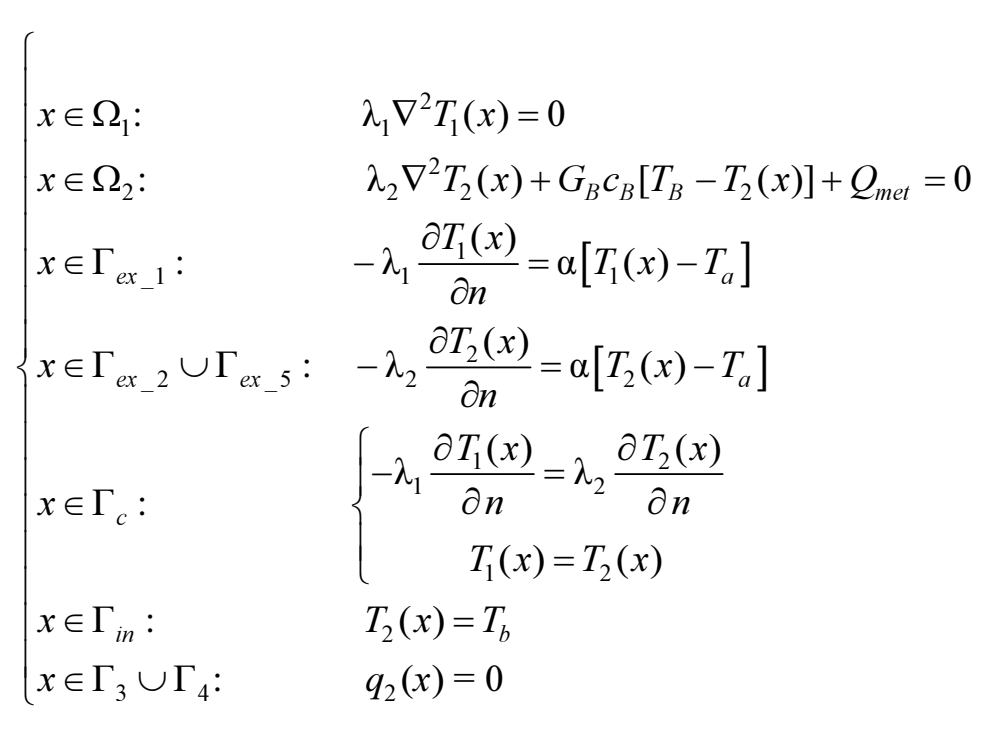

where $x=\left(x_{1}, x_{2}\right)$ are the spatial coordinates, $\lambda_{1}$ is the thermal conductivity of burned tissue, $\lambda_{2}$ is the tissue thermal conductivity, $T_{e}(x)$ is the temperature, $\partial T_{e} / \partial n$ denotes the normal derivative $(e=1,2)$ and $n=\left[\cos \alpha_{1}, \cos \alpha_{2}\right]$ is the normal outward vector, $G_{B}$ is the blood perfusion rate, $c_{B}$ is the specific heat of blood, $T_{B}$ is the arterial blood temperature, $Q_{m e t}$ is the metabolic heat source, $T_{a}$ is the ambient temperature, $\alpha$ is the heat transfer coefficient, $T_{b}$ denotes the known body core temperature and $q_{2}(x)$ is the heat flux.

The position of boundary between burned and healthy tissue $\Gamma_{\mathrm{c}}$ is represented by the Non-Uniform Rational B-Splines (NURBS) curve.

A $n$-th degree NURBS curve is defined as [5]

$$
C(t)=\frac{\sum_{j=0}^{r} N_{j, n}(t) w_{j} \mathbf{P}_{j}}{\sum_{k=0}^{r} N_{k, n}(t) w_{k}}, \quad a \leq t \leq b
$$

where $\mathbf{P}_{j}$ are the control points forming a control polygon, $w_{j}$ are the weights and $N_{j, n}(t)$ are the B-spline basis functions

$$
\begin{gathered}
N_{j, 0}(t)= \begin{cases}1, & t_{j} \leq t \leq t_{j+1} \\
0, & \text { otherwise }\end{cases} \\
N_{j, n}(t)=\frac{t-t_{j}}{t_{j+n}-t_{j}} N_{j, n-1}(t)+\frac{t_{j+n+1}-t}{t_{j+n+1}-t_{j+1}} N_{j+1, n-1}(t)
\end{gathered}
$$


defined for the set of nodes

$$
T=\left\{a, \ldots, a, t_{n+1}, t_{n+2}, \ldots, t_{m-(n+1)}, b, \ldots, b\right\}
$$

at the same time the values $a$ and $b$ appear $n+1$ times. The number of control points equals $r+1$ and corresponds to the number of non-zero basis functions.

\section{Algorithm of boundary elements and internal cells generation}

To determine the temperature field in burned and healthy skin tissue, the boundary element method is used. This method requires the discretization of healthy tissue interior $[6,7]$. In this paper the interior discretization algorithm is presented. The algorithm generates internal cells independently for each sub-domain. It should be pointed out that the coordinates of the boundary nodes located at the common boundaries between sub-domains must be the same. In Figure 2, the correctly generated boundary nodes and internal cells are shown, wherein the double, triple etc. nodes appear, which belong to the several sub-domains.

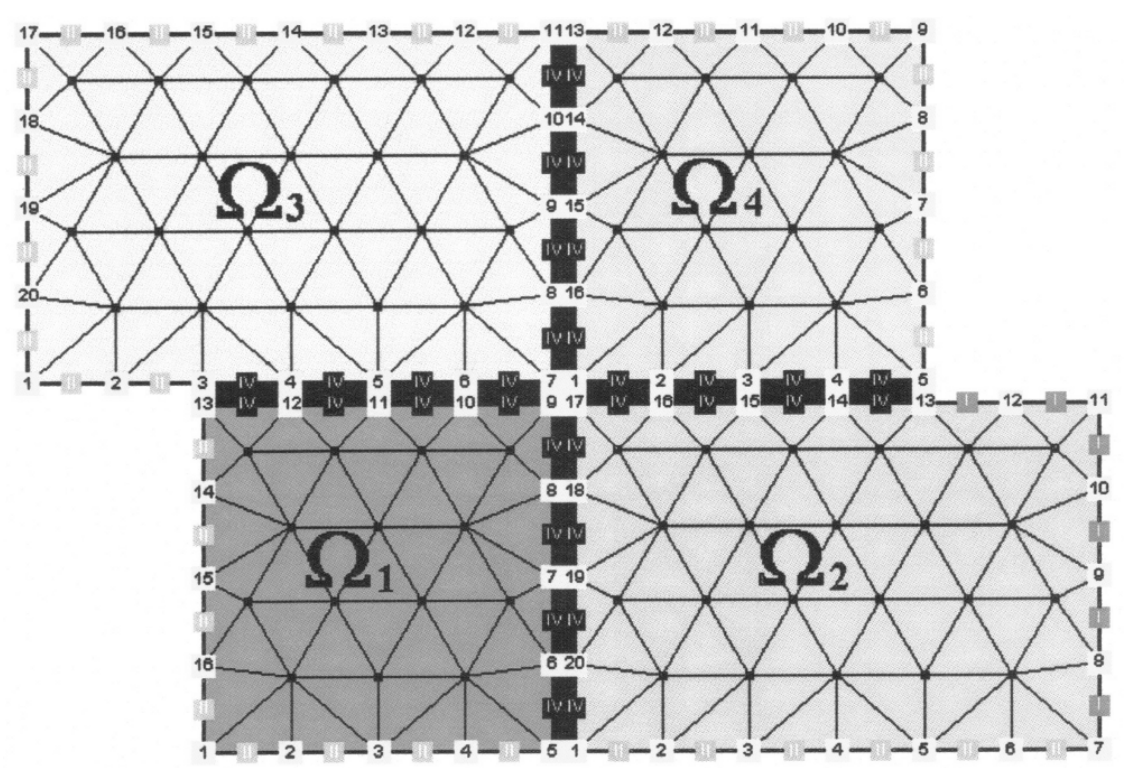

Fig. 2. Contact of sub-domains

If it is possible, we assume the same length of boundary elements on the whole boundary of sub-domains. The domain interior is divided into triangular elements. The triangle side length is equal to the average length of the boundary element $\left(d_{\min }\right)$. The correct discretization of the boundary is essential for discretization of the domain interior. Attention should be paid to the correct discretization of curves 
described by polynomials of higher order, such as NURBS curves. The parametric form of NURBS curves does not allow to divide them into equal parts. So, in this case one should use additional numerical methods which allow one equal division of curve. The algorithm consists of several stages. The first step is to generate the vertices of equilateral triangles. Generation of these elements is made by means of the regular hexagon shown in the Figure 3, where $g_{e}$ equals $d_{\min }$. Next, all of the vertexes are checked to determine if the triangle belongs to the interior of the domain. If so, the triangle is generated, if not, the triangle is not generated (cf. Fig. 4).
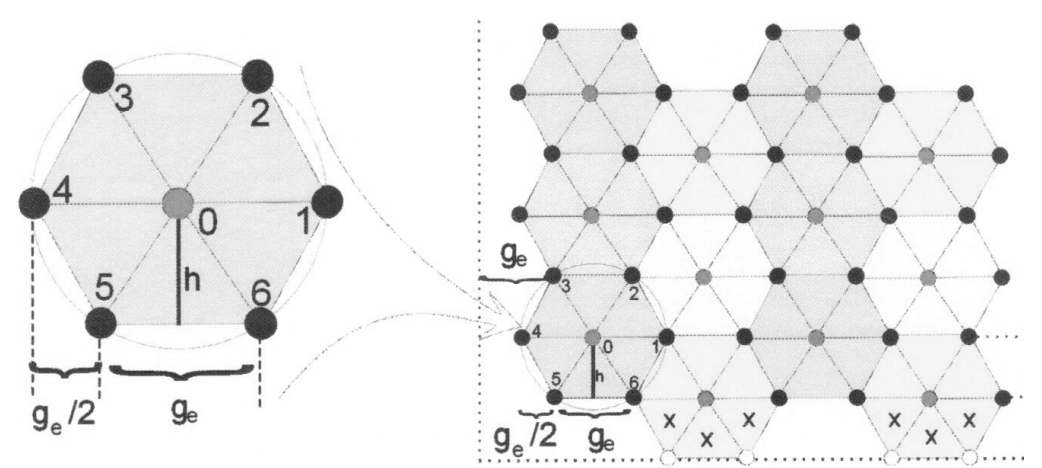

Fig. 3. Generation of internal elements and nodes by means of the regular hexagon

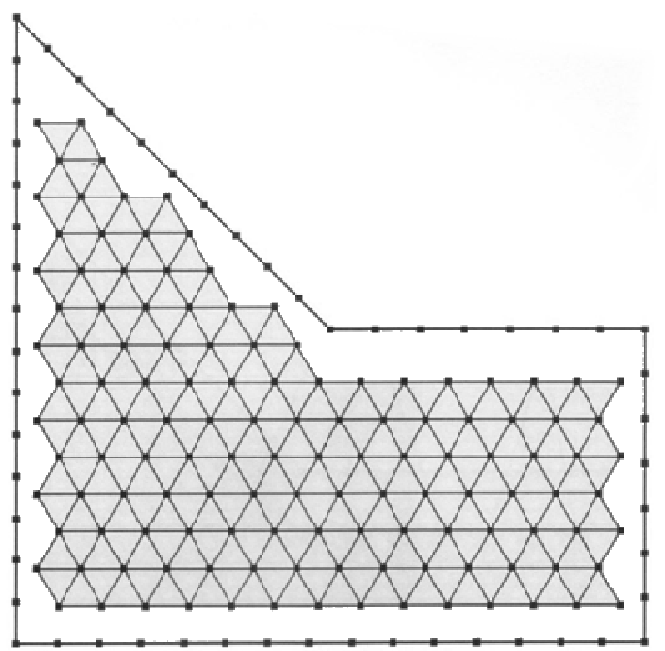

Fig. 4. The first stage of the algorithm

The next step of the algorithm is to search the sides triangles (so-called "external triangles") which are outside of the domain and have been created in the first step. Each side of "external triangle" is connected with the nearest boundary node creating a new triangle (cf. Fig. 5). In the third stage each boundary element is connected with the nearest triangle with the second step. 


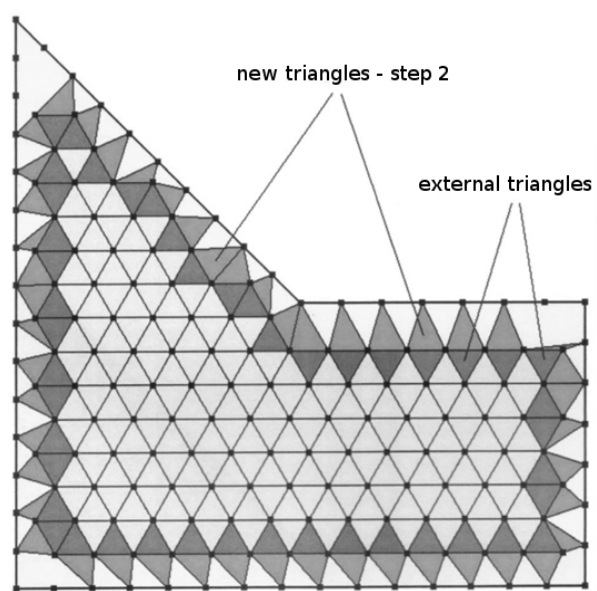

Fig. 5. The second stage of the algorithm

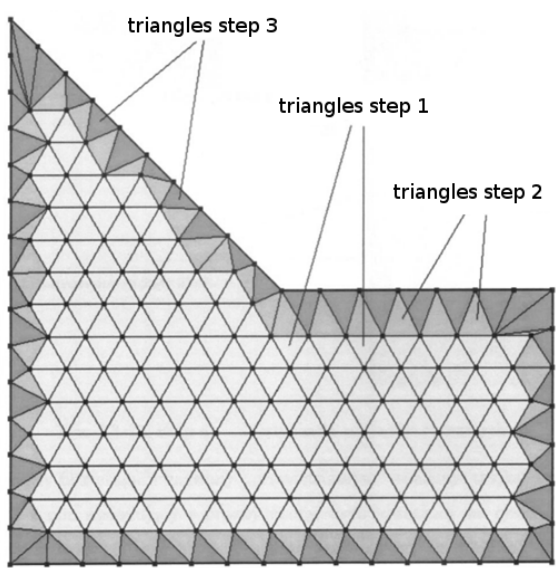

Fig. 6. The third stage of the algorithm

Because the algorithm presented requires the creation of only acute triangles, then the last step is to convert obtuse triangles into acute ones. Details of generation are available in [8].

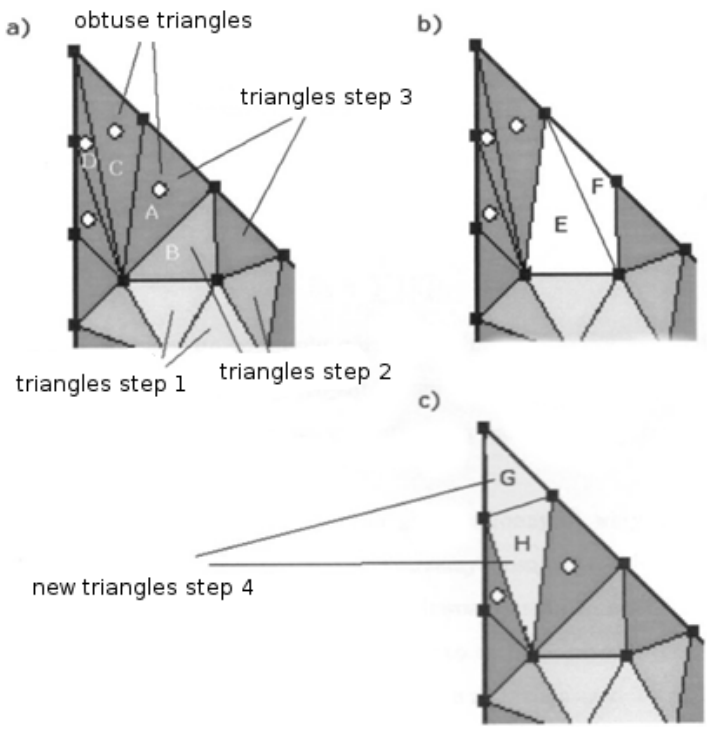

Fig. 7. The last stage of the algorithm

\section{Determination of the temperature field}

The domain of dimensions $0.04 \times 0.02 \mathrm{~m}$ has been considered. It was assumed that: $\lambda_{1}=0.1 \mathrm{~W} /(\mathrm{mK}), \quad \lambda_{2}=0.2 \mathrm{~W} /(\mathrm{mK}), G_{B}=0.5 \mathrm{~kg} /\left(\mathrm{m}^{3} \mathrm{~s}\right), \quad c_{B}=4200 \mathrm{~J} /(\mathrm{kgK})$, $\alpha=10 \mathrm{~W} /\left(\mathrm{m}^{2} \mathrm{~K}\right), T_{a}=20^{\circ} \mathrm{C}, T_{B}=37^{\circ} \mathrm{C}, Q_{m e t}=200 \mathrm{~W} / \mathrm{m}^{3}$. 
At first, the boundary between burned and healthy tissue $\Gamma_{c}$ has been described by the following NURBS curve

$$
\Gamma_{c}(t)=\frac{\left(1-t^{2}\right) w_{0} \mathrm{P}_{0}+2 t(1-t) w_{1} \mathrm{P}_{1}+t^{2} w_{2} \mathrm{P}_{2}}{\left(1-t^{2}\right) w_{0}+2 t(1-t) w_{1}+t^{2} w_{2}}
$$

where $\mathrm{P}_{0}=(0.01,0.02), \mathrm{P}_{1}=(0.03,0.006), \mathrm{P}_{2}=(0.03,0.02), w_{0}=1, w_{1}=0.3, w_{2}=1$. Sub-domains have been divided into different linear boundary elements and internal cells (cf. Tab. 1).

In Figures 8-10 the discretization of boundaries and interior $\Omega_{2}$ is shown. The field of the temperature for case D1 is presented in Figure 11.

Figure 12 illustrates the temperature distribution on the external surface for a different number of boundary elements and internal cells.

Number of boundary elements and internal cells

Table 1

\begin{tabular}{|l|l|l|l|}
\hline $\begin{array}{l}\text { Density of } \\
\text { discretization }\end{array}$ & Sub-domain & $\begin{array}{l}\text { Number of boundary } \\
\text { elements }\end{array}$ & $\begin{array}{l}\text { Number of internal } \\
\text { elements }\end{array}$ \\
\hline D1 & $\Omega 1$ & 20 & 0 \\
\hline D2 & $\Omega 2$ & 60 & 398 \\
\hline & $\Omega 1$ & 40 & 0 \\
\hline D3 & $\Omega 2$ & 120 & 1620 \\
\hline & $\Omega 1$ & 60 & 0 \\
\hline & $\Omega 2$ & 180 & 3716 \\
\hline
\end{tabular}

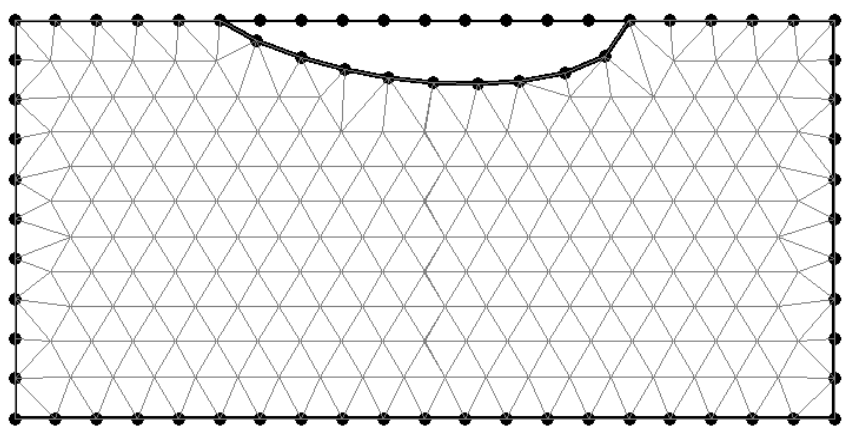

Fig. 8. Discretization of boundaries and interior $\Omega_{2}$ (case D1)

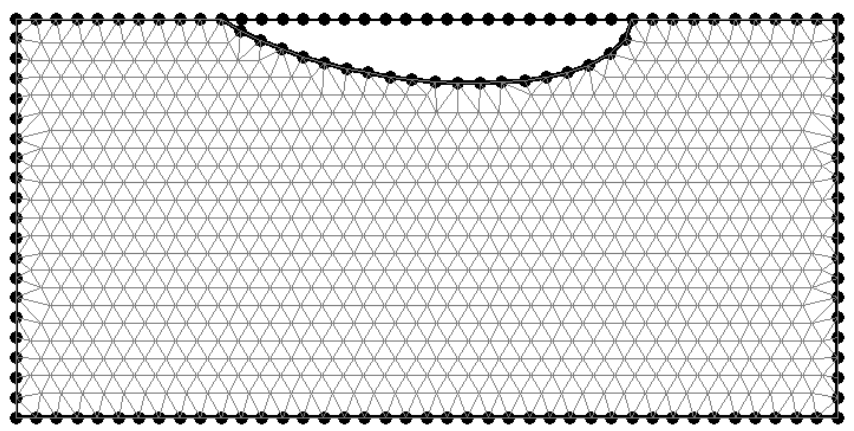

Fig. 9. Discretization of boundaries and interior $\Omega_{2}$ (case D2) 


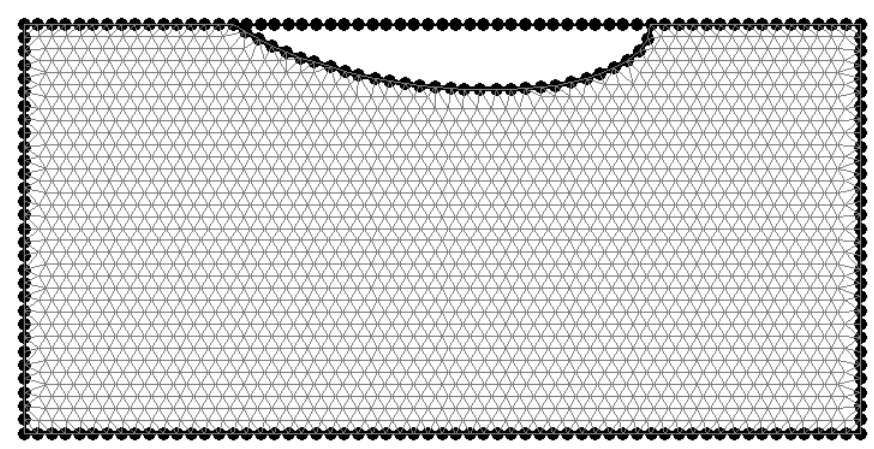

Fig. 10. Discretization of boundaries and interior $\Omega_{2}$ (case D3)

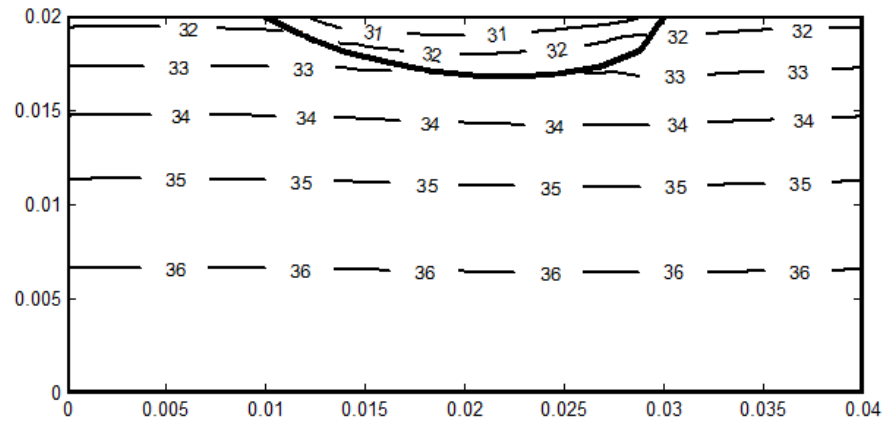

Fig. 11. Temperature distribution for case D1

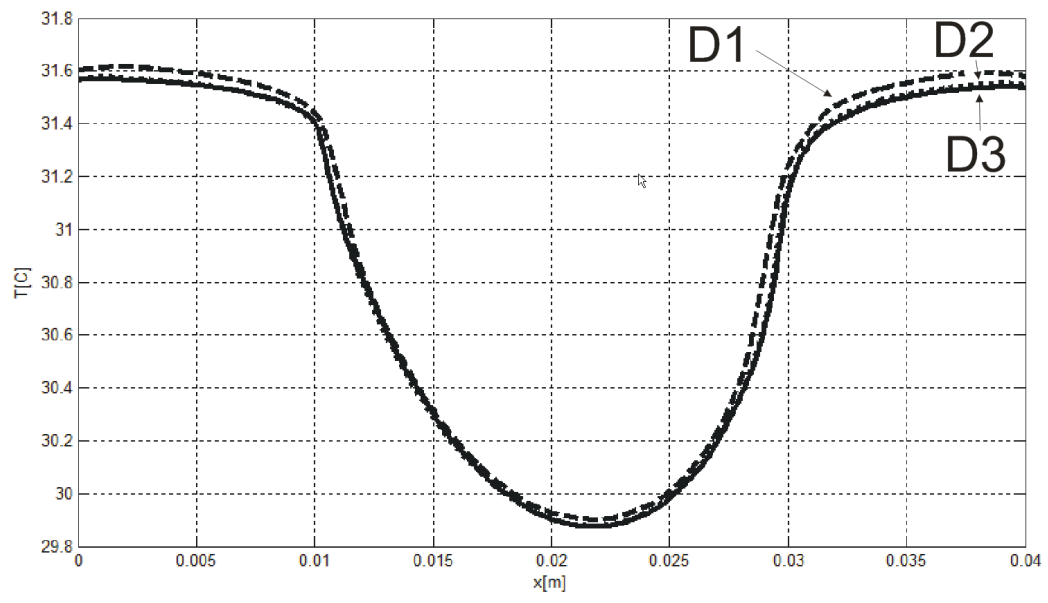

Fig. 12. Temperature distribution on the external surface for different number of boundary elements and internal cells

In all of the cases presented here, the results of calculations are very close. Summing up, using the boundary element method, both the number of boundary elements and internal cells does not have to be too high to ensure high accuracy of the results. 


\section{Acknowledgement}

The article and research are financed within the project N R13 012410 sponsored by the Polish National Centre for Research and Development.

\section{References}

[1] Freus K., Freus S., Majchrzak E., Determination of temperature field at burned and healthy skin tissue using the Boundary Element Method - part I, Journal of Applied Mathematics and Computational Mechanics 2013, 3(12), 39-46.

[2] Pennes H.H., Analysis of tissue and arterial blood temperatures in the resting human forearm, Journal of Applied Physiology 1948, 1, 93-122.

[3] Romero Mendez R., Jimenez-Lozano J.N., Sen M., Gonzalez F.J., Analytical solution of a Pennes equation for burn-depth determination from infrared thermographs, Mathematical Medicine and Biology 2010, 27, 21-38.

[4] Majchrzak E., Dziewoński M., Nowak M., Kawecki M., Bachorz M., Kowalski P., The design of a system for assisting burn and chronic wound diagnosis, [in:] E. Piętka, J. Kawa (eds.), ITIB 2012, LNCS 7339, Springer-Verlag, Berlin-Heidelberg 2012, 110-117.

[5] Piegl L., Tiller W., The NURBS Book, Springer, 1995.

[6] Brebbia C.A., Dominguez J., Boundary Elements. An Introductory Course, CMP, McGraw-Hill Book Company, London 1992.

[7] Majchrzak E., Boundary Element Method in Heat Transfer, Publ. of the Techn. Univ. of Czest., Czestochowa 2001 (in Polish).

[8] Freus S., Doctoral thesis, Czestochowa University of Technology, 2003. 